\title{
Plasma Jet-Substrate Interaction in Low Pressure Plasma Spray-CVD Processes
}

\author{
Ph. Guittienne, D. Grange, Ch. Hollenstein, and M. Gindrat
}

(Submitted April 7, 2011; in revised form October 17, 2011)

\begin{abstract}
Conventional equipment for plasma spraying can be adapted for operation at low pressure so that PECVD-like processing can be performed. The plasma jet generated by the torch is characterized by a high convective velocity and a high gas temperature. The influence of these properties on a deposition process are investigated in the framework of simple theoretical considerations and illustrated by various experimental results obtained with $\mathrm{SiO}_{x}$ deposition. A conclusion of this study is that the deposition process is dominated by diffusion effects on the substrate surface: the deposition profiles and the deposition rates are determined by the precursor density and by the gas temperature on the substrate surface. The high velocity of the jet does not play a direct role in the deposition mechanism. On the other hand it strongly increases the precursor density available for the deposition since it efficiently transports the precursor up to the substrate.
\end{abstract}

Keywords coating, heat transfer, low pressure, PECVD, plasma torch, thin film

\section{Introduction}

Plasma based processes are well known to be very widely used in many industrial applications, and for the production of very various types of products. Quite popular examples of plasmas applications are silicon etching, typically for integrated circuits (IC) manufacturing, and silicon or transparent conductive oxides (TCO) thin film deposition for solar cell production. But plasmas are also encountered in food packaging industry, space propulsion thrusters, compact discs manufacturing, flat screen televisions, waste treatment technology, and many others. As a consequence of these competitive markets interest there is a constant demand for the concerned industries in processes performance improvement. Within the specific needs inherent to each application two general trends can be distinguished: faster and larger. Especially large area processing is of great interest as it could allow a larger amount of work pieces to be treated at the same time, but also new kinds of products to be developed as larger work pieces could be processed.

A large part of plasma based processes concerns thin film deposition (typ. $100 \mathrm{~nm}$ ) which is currently obtained by plasma-enhanced chemical vapour deposition (PEC-

Ph. Guittienne, D. Grange, and Ch. Hollenstein, Centre for Research in Plasma Physics (CRPP), Ecole Polytechnique Fédérale de Lausanne (EPFL), Lausanne, Switzerland; and M. Gindrat, Sulzer Metco Switzerland AG, Wohlen, Switzerland. Contact e-mails: christophe.hollenstein@epfl.ch and david.grange@ epfl.ch.
VD) typically performed under low pressure conditions (1-100 Pa) (Ref 1). A major part of the PECVD reactors relies on radio frequency (RF) capacitive or inductive sources, but difficulties are encountered in the attempt to obtain large and fast reactors.

In a totally other direction thermal plasma spraying (Ref 2) was developed to provide rapid, thick and porous coatings (typ. $1 \mathrm{~mm}$ ). Thanks to the high enthalpy and the high convective velocity of the plasma jet created by a DC torch, powders of desired materials can be injected and melted into the jet and then convectively transported up to the substrate on which a coating is formed by accumulation of splats. These processes are typically performed under high pressure conditions $\left(10^{4}-10^{5} \mathrm{~Pa}\right)$.

The use of thermal plasmas for dense coating production was already envisaged in the early 1990s (Ref 3). In a first time low pressure (typ. $10^{4} \mathrm{~Pa}$ ) processes were developed the injection of micrometric powders allowing dense coatings (typ. $10 \mu \mathrm{m}$ thick) at high deposition rates (Ref 4-6). Then, by modifying conventional thermal spray components in order that the torch can be operated under very low pressures (typ. $50 \mathrm{~Pa}$ ) a new process allowing CVD-like coatings $(1-100 \mathrm{~nm})$ to be produced at high deposition rates (Ref 7) has been developed. This PSCVD (plasma spray-CVD) technique has been shown to be efficient for various thin film deposition processes (pure metal, metal oxides, a-CH, $\mathrm{SiO}_{x}$ ) but also for surface modifications (carburisation or nitruration of zirconia pieces). The ability of PSCVD to provide fast processes is essentially due to the high dissociation potency of the plasma jet which allows high precursor fluxes to be efficiently used. Moreover a great potential of this technique is in the possibility of combined processes associating CVD and conventional spraying.

To conclude with the specificities of PSCVD, it could be noted that under very low pressures the diameter of the plasma jet is large (typ. $20 \mathrm{~cm}$ ) and that plasma torches are 


\begin{tabular}{|c|c|c|c|}
\hline \multicolumn{4}{|c|}{ Nomenclature } \\
\hline$n_{\mathrm{g}}$ & Total particle density & $\xi_{\mathrm{T}}$ & Particle thermalization efficiency \\
\hline$T$ & Gas temperature & $D$ & Mass diffusivity \\
\hline$U_{0}$ & Initial convective velocity & $k$ & Fluid thermal conductivity \\
\hline$n_{\mathrm{p}}$ & Precursor density & $C_{\mathrm{g}}$ & Fluid specific heat \\
\hline$\lambda$ & Particle mean free path & $\rho_{\mathrm{g}}$ & Fluid mass density \\
\hline$V_{\text {th }}$ & Thermal velocity & $T_{\mathrm{s}}$ & Substrate temperature \\
\hline$k_{\mathrm{B}}$ & Boltzmann constant $=1.38 \times 10^{-23} \mathrm{~J} \mathrm{~K}^{-1}$ & $\rho_{\mathrm{s}}$ & Substrate mass density \\
\hline$m_{\mathrm{p}}$ & Precursor particles mass & $C_{\mathrm{s}}$ & Substrate specific heat \\
\hline$\phi_{\mathrm{N}}$ & $\begin{array}{l}\text { Averaged particle flux oriented in a given } \\
\text { direction }\end{array}$ & $d$ & Substrate thickness \\
\hline \multirow{2}{*}{$\begin{array}{l}\xi \\
\phi_{\mathrm{D}}\end{array}$} & Deposition efficiency & \multicolumn{2}{|r|}{ Abbreviations } \\
\hline & to the coating growth & $\mathrm{IC}$ & Integrated circuit \\
\hline$\eta_{D}$ & Deposition rate & TCO & Transparent conductive oxide \\
\hline$\rho_{\mathrm{D}}$ & Coating mass density & PECVD & Plasma-enhanced chemical vapour deposition \\
\hline$T_{0}$ & Initial gas temperature & $\mathrm{RF}$ & Radio frequency \\
\hline$n_{0}$ & Initial precursor density & $\mathrm{DC}$ & Direct current \\
\hline$\phi_{\mathrm{g}}$ & Normal neutral flux & CVD & Chemical vapour deposition \\
\hline$\Delta E$ & Statistical energy variation & PSCVD & Plasma spray chemical vapour deposition \\
\hline$m_{\mathrm{g}}$ & Mass of a neutral & $\mathrm{a}-\mathrm{CH}$ & Amorphous hydrogenated carbon \\
\hline$Q_{\max }$ & Maximum heat flux & HMDSO & Hexamethyldisiloxane \\
\hline$Q$ & Effective heat flux & & \\
\hline
\end{tabular}

currently mounted on multi-axis displacement system. It then appears that PSCVD could be a very interesting candidate for some large area processing.

In comparison with conventional PECVD systems, based on sustained glow discharges, the PSCVD obviously presents some major differences: the plasma jet is approximately thermalized (electrons, ions and neutrals have the same temperature), the gas temperature into the jet is very high (typ. $1000 \mathrm{~K}$ in the vicinity of the substrate) and finally the plasma impinges on the substrate with a very high convective axial velocity (typ. $500 \mathrm{~m} / \mathrm{s}$ ) (Ref 7). The question then arises as how, and in what proportions, these specificities affect the CVD processes. We can expect the thermalization of the plasma to have mainly some consequences on the process chemistry, but this aspect is beyond the scope of this article. We focus here on the impact of the high convective velocity and of the high temperature of the plasma jet on the coating formation. In a first step theoretical considerations will be introduced to figure out what are the relevant parameters with regard to the deposition mechanisms and how these can be affected by the jet velocity and temperature. Then a selection of experimental results will be presented and analysed according to this theoretical approach.

\section{Theoretical Approach of the Jet-Substrate Interaction}

The system considered here can be sketched as shown in Fig. 1: the plasma jet impinging on a substrate is characterized by its total particle density $n_{\mathrm{g}}$, its gas temperature $T$, its radial velocity $\left(U_{0}(r)\right)$ profile, far upstream with regards to the substrate, and by a precursor density $n_{\mathrm{p}}$ generally much smaller than $n_{\mathrm{g}}$ (typ. $n_{\mathrm{p}} / n_{\mathrm{g}} \sim 1 \%$ ). For a deposition to occur on the substrate the precursor particles must impact its surface. In a thermal spray process the droplets of melted material are transported convectively by the gaseous fluid toward the substrate surface and, thanks to their inertia, impact it ballistically. The situation is very different when we deal with molecular or atomic precursors which are constituents of the fluid itself (Ref 1, $8,9)$. If we were to consider the precursor transport in the framework of a purely convective model, the normal component of the fluid velocity on the substrate being strictly equal to zero, the surface would not receive any flux (Ref 10). To understand the origin of the precursor particle flux on the substrate we have to consider what happens in the vicinity of its surface, on a spatial scale smaller than the particle mean free path $\lambda$. On this scale the particle density stills well defined provided that $\lambda \gg n_{\mathrm{g}}{ }^{-1 / 3}$, which is generally the case (typ. $\lambda \sim 1 \mathrm{~mm}$ and $n_{\mathrm{g}}{ }^{-1 / 3} \sim 0.1 \mu \mathrm{m}$ for a $10 \mathrm{~Pa}$ pressure and a gas temperature of $1000 \mathrm{~K}$ ). So every particle can be taken to move at the thermal velocity $V_{\mathrm{th}}=\sqrt{\frac{8 k_{\mathrm{B}} T}{\pi m_{\mathrm{p}}}}(\operatorname{Ref} 1)$ in any direction with the same probability ( $m_{\mathrm{p}}$ is precursor particle mass).

According to the kinetic theory, the averaged particle flux $\phi_{\mathrm{N}}$ oriented in a given direction, and a fortiori toward the substrate surface, can be taken to be (Ref $1,8,9)$ :

$$
\phi_{\mathrm{N}}=\frac{n_{\mathrm{p}} V_{\mathrm{th}}}{4}
$$

This expression corresponds to the maximum flux that could contribute to a coating formation. But obviously some of the colliding particles may not stick on the substrate for reasons linked for example to the substrate

\section{.}




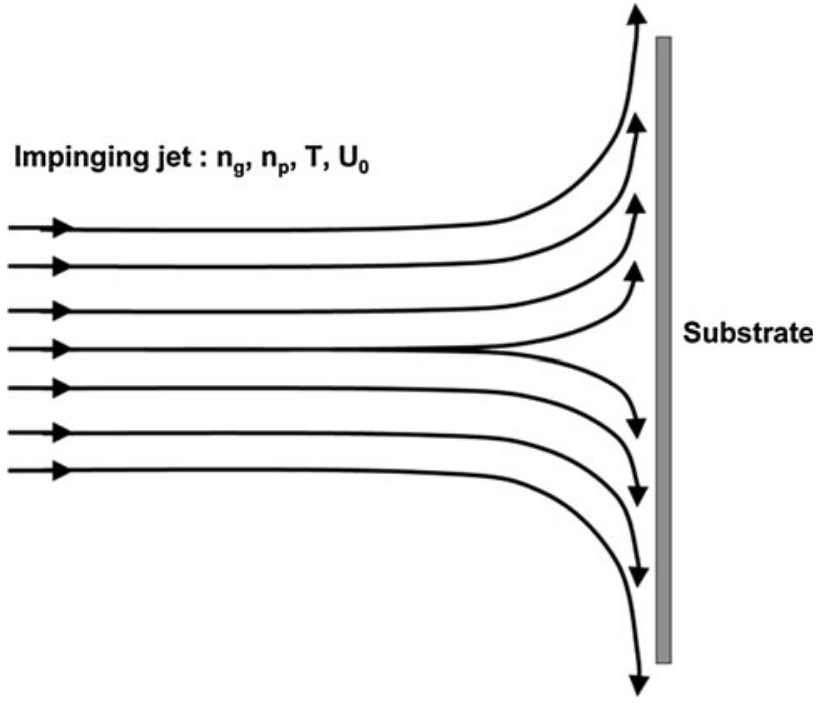

Fig. 1 Impinging jet; the lines figurate the fluid streamlines

temperature or to the process chemistry. All these influences can be taken into account by introducing a deposition efficiency, or sticking coefficient, $\xi$ (Ref 11) so that the normal flux $\phi_{\mathrm{D}}$ effectively contributing to the coating growth is finally given by:

$$
\phi_{\mathrm{D}}=\xi \phi_{\mathrm{N}}=\xi \frac{n_{\mathrm{p}} V_{\mathrm{th}}}{4}
$$

The deposition rate $\eta_{\mathrm{D}}$ can be related to $\phi_{\mathrm{D}}$ as follows: $\eta_{\mathrm{D}}=\frac{\phi_{\mathrm{D}} m_{\mathrm{p}}}{\rho_{\mathrm{D}}}$, with $\rho_{\mathrm{D}}$ the coating mass density.

It has to be said that the mass flux $m_{\mathrm{p}} \phi_{\mathrm{D}}$ associated to the deposition process is responsible for a convective flux on the substrate surface known as the Stephan flow (Ref 9). In the present case the Stephan velocity can be estimated to be $0.25 \mathrm{~m} \mathrm{~s}^{-1}$, and can then be neglected with regard to the particle thermal velocity (typ. $750 \mathrm{~m} \mathrm{~s}^{-1}$ ).

If the fluidic velocity doesn't play a direct role in the deposition mechanism, which depends only on the substrate surface conditions, it will on the other hand strongly affect these conditions. To illustrate this point a simplified 1D model of the impinging jet problem is considered, as sketched in Fig. 2. At $z=0$ the jet initial conditions are defined: convective velocity $U_{0}$, gas temperature $T_{0}$ and precursor density $n_{0}$. In the whole system the total neutral density $n_{\mathrm{g}}$ is taken to be constant, or in other words a constant pressure is imposed. In the region comprised between $z=0$ and $z=L$ (substrate) the fluid velocity is set to decrease linearly from $U_{0}$ to 0 . The second region between $z=L$ and $z=L+L 2$ is non convective and is only required to impose a second isotherm, taken here to be the ambient temperature. On the left side of the $z=L$ interface, the substrate pumps a deposition flux $\phi_{\mathrm{D}}$, noting that in the following the maximum deposition efficiency $\xi$ is taken. Heat transfers occur on both sides of the interface and the corresponding heat fluxes can be approximated according to the following reasoning. The heat transfer occurs essentially by means of the neutral-substrate interaction. Taking the gas temperature to be $T_{1}$

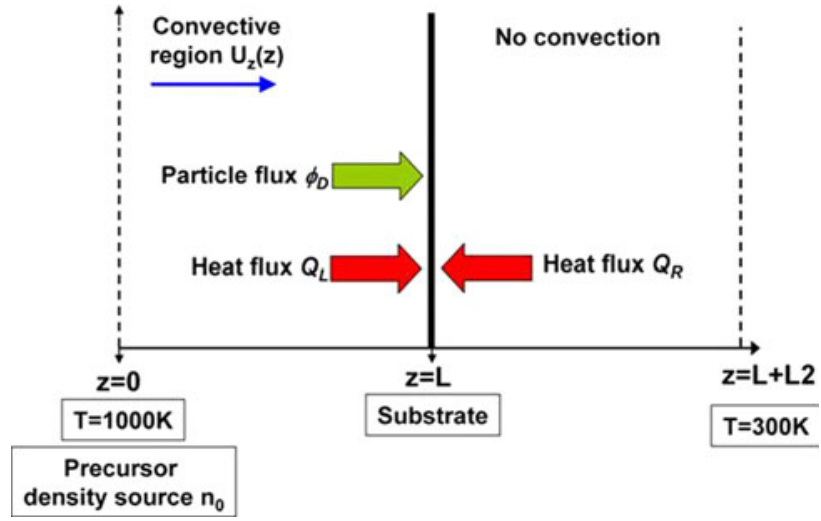

Fig. 2 1D model

for a given side of the interface, the latter receives a normal neutral flux $\phi_{\mathrm{g}}$ given by $(\operatorname{Ref} 1,8,9)$ :

$$
\phi_{\mathrm{g}}=\frac{n_{\mathrm{g}} V_{\mathrm{th} 1}}{4}
$$

For each particle-surface interaction the neutral can take or give a certain amount of energy which depends on the inelasticity of the collisions. The maximum energy transfer would correspond to a thermalization of the particle with the substrate. If the substrate temperature is taken to be $T_{2}$, the thermalization process would then involve a statistical energy variation $\Delta E$ that can be expressed as:

$$
\Delta E=\frac{1}{2} m_{\mathrm{g}}\left(V_{\mathrm{th} 1}^{2}-V_{\mathrm{th} 2}^{2}\right),
$$

with $m_{\mathrm{g}}$ the mass of a neutral.

If this thermalization is applied to the total incident neutral normal flux, the maximum heat flux $Q_{\max }$ that can be transmitted at the interface is obtained:

$$
Q_{\max }=\phi_{\mathrm{g}} \Delta E=\frac{k_{\mathrm{B}} n_{\mathrm{g}} V_{\mathrm{th} 1}}{\pi}\left(T_{1}-T_{2}\right)
$$

The inelasticity of the collision processes can then be characterized by introducing a particle thermalization efficiency $\xi_{\mathrm{T}} \in[0,1]$ such as the effective heat flux $Q$ on the substrate is given by:

$$
Q=\xi_{\mathrm{T}} Q_{\max }
$$

Once all the boundary conditions are set the precursor density profile $n_{\mathrm{p}}(z)$ and the gas temperature profile $T(z)$ can be analytically calculated by solving respectively the convection-diffusion equation and the conduction-convection equation, which can be written in the approximation of an incompressible flow as ( Ref 8):

$$
\left\{\begin{array}{r}
-D \vec{\nabla} \cdot \vec{\nabla} n_{\mathrm{p}}+\vec{U}_{\mathrm{z}} \cdot \vec{\nabla} n_{\mathrm{p}}=0 \\
-k \vec{\nabla} \cdot \vec{\nabla} T+\rho_{\mathrm{g}} C_{\mathrm{g}} \vec{U}_{\mathrm{z}} \cdot \vec{\nabla} T=0
\end{array}\right.
$$

Here $D$ is the precursor diffusivity (taken to be constant), $k$ is the fluid thermal conductivity, $C_{\mathrm{g}}$ is the fluid specific heat and $\rho_{\mathrm{g}}=n_{\mathrm{g}} m_{\mathrm{g}}$ is the fluid mass density. Figure 3 shows the evolution of the ratio $n_{\mathrm{p}}(z) / n_{0}$ for three different values of the fluid initial velocity $U_{0}$, a value of $500 \mathrm{~m} / \mathrm{s}$ 


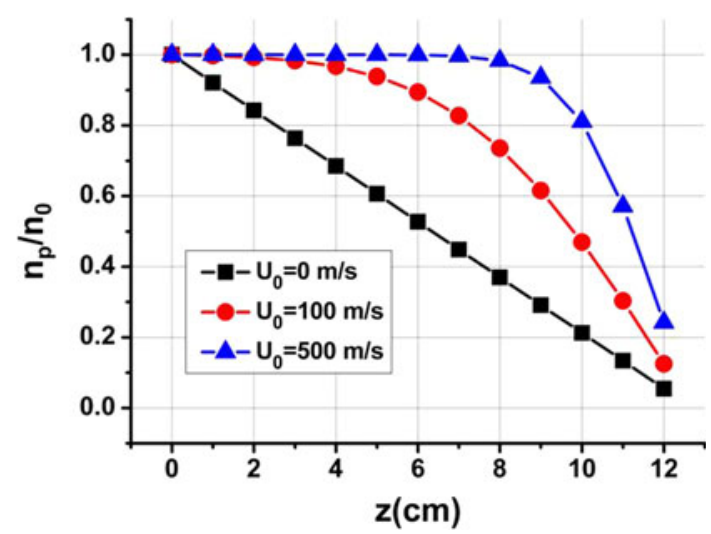

Fig. 3 Precursor density profiles $(\xi=1)$ for $U_{0}=0,100$, and $500 \mathrm{~m} / \mathrm{s}$

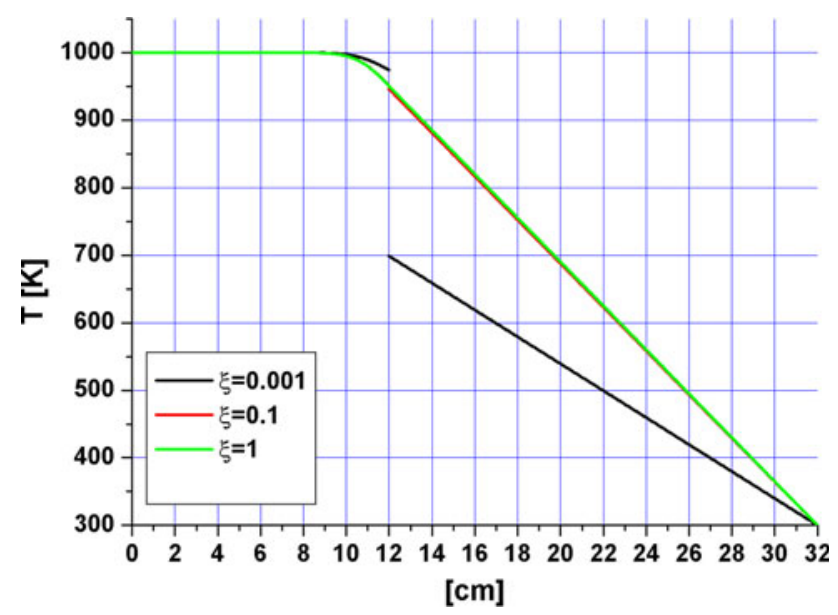

Fig. 4 Gas temperature profiles for $\xi_{\mathrm{T}}=(0.001,0.1,1)$ with $\mathrm{U}_{0}=500 \mathrm{~m} / \mathrm{s}$

being comparable to our experimental conditions. It can be seen in Fig. 3 that the precursor density, and therefore the deposition rate, can be increased by a factor 5 when the fluid velocity rises from zero to $500 \mathrm{~m} / \mathrm{s}$. Figure 4 shows the typical temperature profiles obtained for different values of the thermalization efficiency $\xi_{\mathrm{T}}$, calculated for an initial fluid velocity of $10 \mathrm{~m} / \mathrm{s}$, while Fig. 5 shows the gas temperature on the deposition surface (left side of the interface) as a function of the initial velocity $U_{0}$. It has first to be noticed that the value of the thermalization efficiency does not affect much the gas temperature on the deposition side of the interface, which means that it is not a key parameter with regards to the maximum deposition rate $\phi_{\mathrm{N}}$. The temperature jump observed at the interface is a consequence of the definition of the approximated heat fluxes given above. It can be seen that as long as $\xi_{\mathrm{T}}$ is large enough (typ. for $\xi_{\mathrm{T}}>0.01$ ) the jump amplitude is small and the temperature profile is very close from what would be obtained in the framework of a continuous model. Secondly we observe that the gas temperature on the interface increases very rapidly with $U_{0}$ and reach almost a saturation value close to $T_{\mathrm{p}}$ at only $10 \mathrm{~m} / \mathrm{s}$.

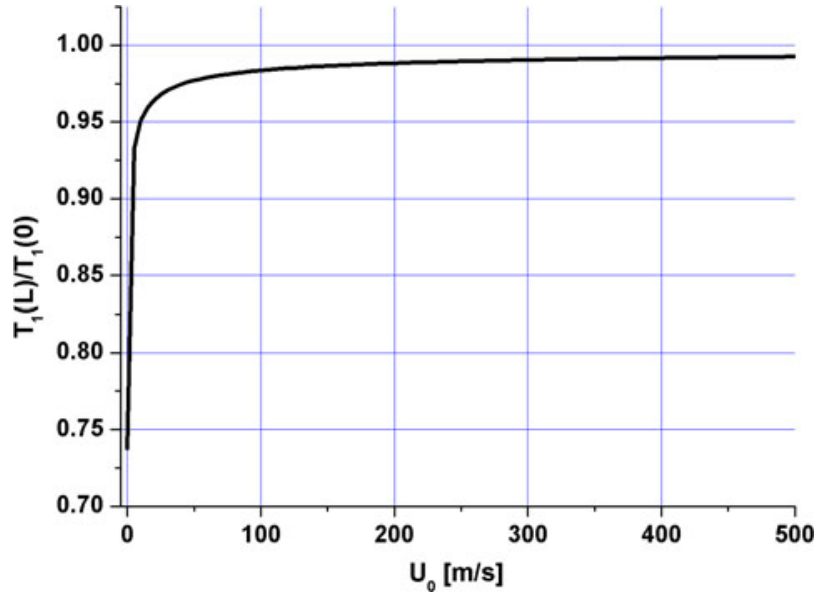

Fig. 5 Normalized gas temperature on the left side of the substrate as a function of the initial fluid velocity $\mathrm{U}_{0}$

To resume our theoretical approach results, it appears that the high velocity of the plasma jet should not play any role in the deposition mechanism itself, but its influence on the deposition rate can nevertheless be very important as the convective transport of the precursor from the source to the substrate strongly increases the density $n_{\mathrm{p}}$ available for the coating growth. The gas temperature plays a secondary role compared to the precursor concentration, at first because of its square root dependence in the expression of the maximum deposition rate $\phi_{\mathrm{N}}$, and secondly because the heat convective transport due to the jet velocity does not affect very much the gas temperature on the substrate. With regard to the maximum deposition rate value $\phi_{\mathrm{N}}$ the high velocity of the jet and its high temperature are positive points playing in favour of the PSCVD system. On another hand it will be showed that the high gas temperature can be counter productive with regard to the deposition efficiency $\xi$ because this one is lowered when the substrate temperature increases. Finally, the gas pressure has been taken to be constant in our 1D model. It will be shown in the next section that in reality the impinging jet creates a higher pressure zone in front of the substrate. Then the impact of the jet velocity on the deposition rate and on the heat fluxes is not only limited to transport phenomena but also comprises an enhancement of the gas and precursor densities on the substrate surface due to the local pressure increase.

\section{Experimental Results}

A set of experimental results is presented here that illustrate some expected phenomena according to our theoretical approach. All the experiments shown here were performed with $\mathrm{SiO}_{x}$ depositions (Ref 7). The argon + oxygen plasma jet is injected into a confinement tube, in order to minimize recirculation-associated phenomena (Fig. 6). The argon flow rate is typically of about 30 standard liters per minute (SLPM) while the oxygen 


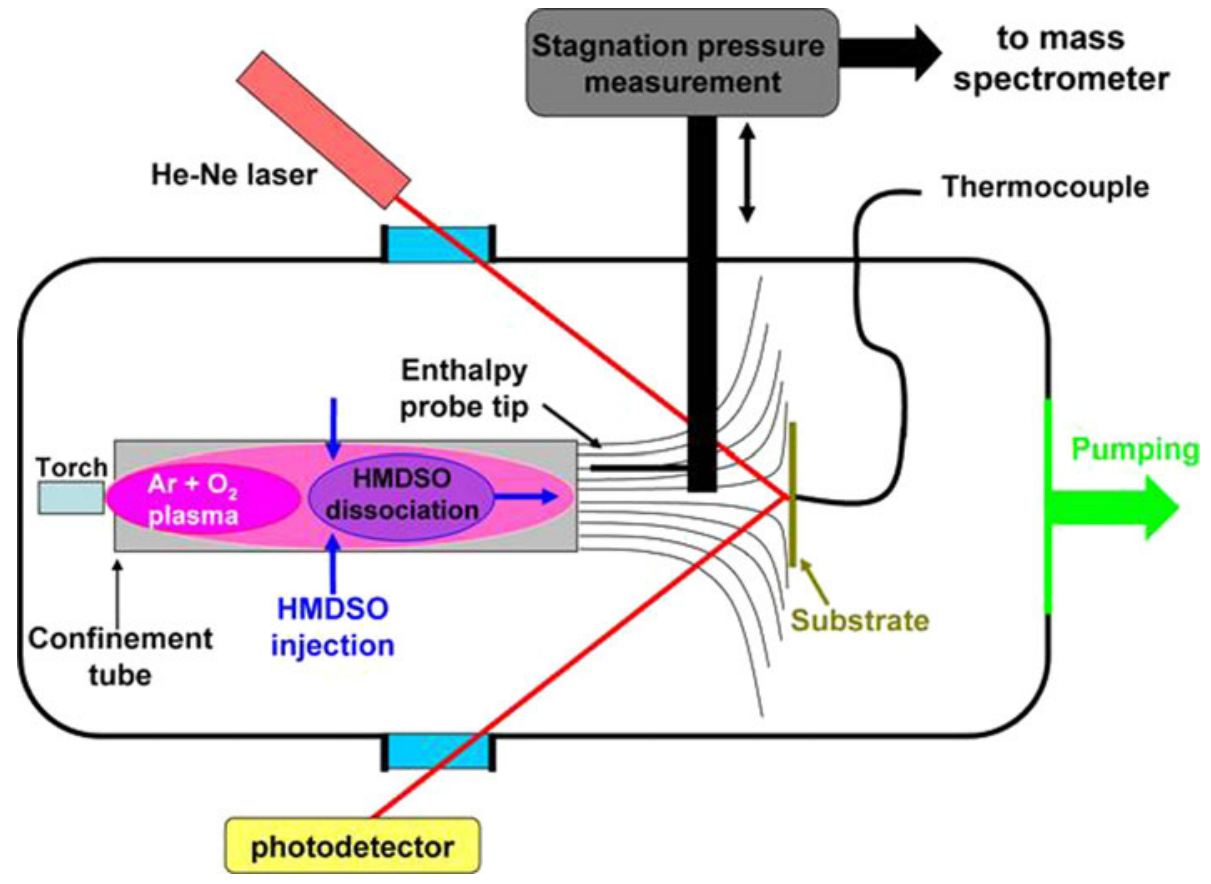

Fig. 6 Experimental setup

flow rate is of about 2 SLPM. The Si precursor is obtained by dissociation of evaporated hexamethyldisiloxane (HMDSO) injected inside the confinement tube through a perforated ring. The typical evaporation rate for the HMDSO is $30 \mathrm{~g} / \mathrm{h}$ which corresponds to a gaseous flow rate of about 0.07 SLPM. An enthalpy probe system (Ref 12) was used to estimate the fluid velocity profile at the confinement tube outlet. A typical value of $550 \mathrm{~m} / \mathrm{s}$ was found on the jet axis. The enthalpy probe was also used to sample the plasma in order to perform some mass spectroscopy measurements. The gas temperature on the sides of the tube outlet and on the surface of the substrates has been measured by means of thermocouples, as well as the substrate temperature. A typical value of $900 \mathrm{~K}$ was found for the gas temperature on the jet axis in front of the substrate. A He-Ne $(632 \mathrm{~nm})$ laser can be pointed on the substrate surface in order to perform in situ interferometry allowing information on the growing coatings to be obtained.

Figure 7 shows the measurements of the deposition rate profiles (measured by interferometry) on two identical small steel substrates $(7 \times 7 \mathrm{~cm})$. For the first one (Fig. 7a) the substrate was held during the deposition process by a shaft screwed in its rear side, so the deposition surface was perfectly plane. For the second one (Fig. 7b), the steel plate was supported by a substrate holder forming a $3 \mathrm{~mm}$ step on the edges of the sample. The two deposition profiles show completely opposite trends, as for sample (a) the profile rises rapidly on the plate's edges (typ. 40\%) while it drops to zero for sample (b). This result is a good illustration of the fact that the deposition process is dominated by diffusion effects in the vicinity of the substrate surface, even in the present case of a high velocity impinging jet. Furthermore, it is a quite general evidence of the importance of the substrate geometry and environment with regards to the coating uniformity. As a matter of fact the opposite trends of the deposition profiles shown in Fig. 7 can be explained by the discontinuity of the precursor flux pumped by the substrate and its environment in a purely diffusive model.

To illustrate this point, first consider a simple result arising from the 1D model presented in the previous section. In a purely diffusive situation $\left(U_{0}=0\right)$ the precursor density at the interface (substrate) calculated from the convection-diffusion equation is given by:

$$
n_{\mathrm{p}}(L)=\frac{4 D n_{0}}{4 D+\xi V_{\mathrm{th}} L}
$$

It then appears that the precursor density on the substrate is inversely linear proportional to the deposition efficiency $\xi$.

The 2D situation shown in Fig. 8(a) where the interface is divided into two semi-infinite regions is now considered: a first that pumps the deposition flux, which should be identified as the substrate, and another one that doesn't pump any flux. If we look far enough from the transition zone between these two semi-infinite regions, the density on the interface is well known and corresponds to the results of the $1 \mathrm{D}$ problem: in the non-pumping region the density is $n_{0}$, while in the pumping region the density on the interface is much lower (typ. $1 \%$ of $n_{0}$ ). In the transition zone a density profile linking these two extreme solutions is created. A calculation of this density profile is shown on the lower part of Fig. 8(a), while Fig. 8(c) shows a zoom of the density profile on the deposition region. It appears that the density on the edge of the pumping 


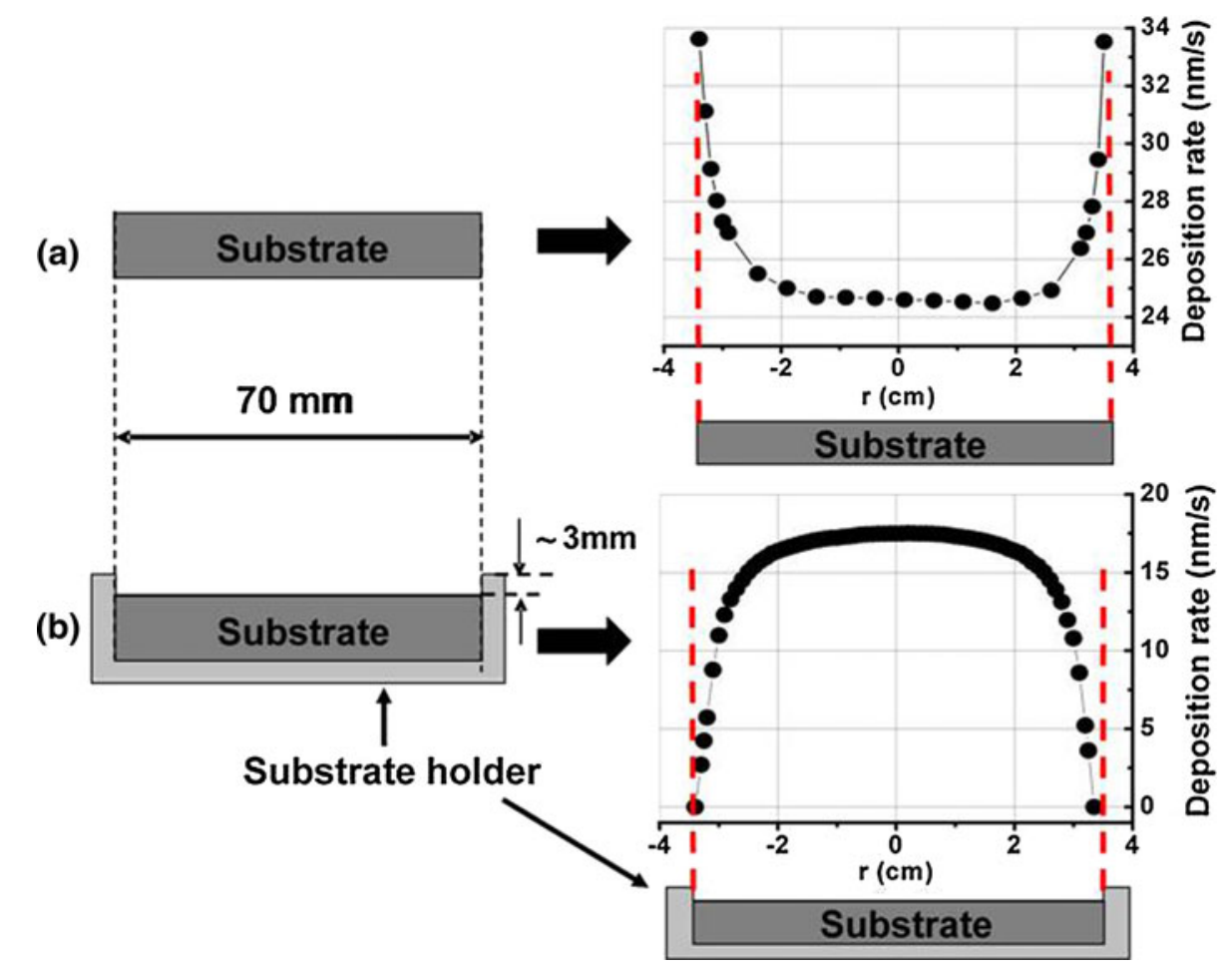

Fig. 7 Deposition rate profiles on small substrates: (a) plane deposition surface and (b) 3 mm step on the deposition surface edges

interface rises under the influence of the non pumping region and this is the origin of the deposition rate profiles obtained on perfectly flat surfaces as the one shown in Fig. 7(a).

Let's now consider the 2D situation sketched in Fig. 8(b) for which a step on the edge of the substrate has been added. Now the step pumps a deposition flux as well as the substrate. As long as we look at the density on the surface far away from the corner, the pumping generated by the step can be neglected and the density is given by the 1D solution. But close to the corner the density is affected by a double pumping and is then strongly lowered compared to the 1D solution. A 2D calculation of the density profile in the vicinity of a pumping corner is shown in the lower part of Fig. 8(b) and it can be seen that the density effectively drops to zero on the substrate edges as does the deposition rate profile shown in Fig. 7(b).

In the previous example the deposition efficiency $\xi$ is taken to be constant over the whole substrate surface and the deposition rate profiles are only affected by the precursor pumping discontinuities. The assumption of a constant value for $\xi$ can reasonably be made because the sample dimensions are much smaller than the jet diameter, which implies notably a uniform heating of the substrates. In fact the deposition efficiency is sensitively dependant on the local substrate temperature, as illustrated by the profiles presented in Fig. 9 which compares the deposition rate profiles obtained on three thin $(1 \mathrm{~mm})$ copper plates differently thermalized: not cooled at all, uniformly cooled and locally cooled (cold point). The profile obtained by creating a local cold point on the copper plate shows that the deposition efficiency is really dependent on the local substrate temperature. On the other hand we observe that as long as the substrate temperature stays uniform the deposition profile is qualitatively unchanged although the mean value of deposition rate is increased by $25 \%$ if the substrate is cooled. In this case the shape of the profile, especially the increase on the edges, is determined by the precursor pumping discontinuities while the temperature of the substrate determines the value of $\xi$, and then the mean deposition rate.

In an attempt to determine the substrate temperature dependence of the deposition efficiency the time evolution of the deposition rate $\eta_{\mathrm{D}}$ during long deposition processes (typ. $500 \mathrm{~s})$ on small $(10 \mathrm{~cm}$ by $10 \mathrm{~cm})$ but thick $(1 \mathrm{~cm})$ copper and aluminium substrates has been measured. To do so time resolved interferometry $(632 \mathrm{~nm})$ has been used which allows an in situ determination of the coating thickness during the deposition (Fig. 6). At the same time the evolution of the substrate temperature $T_{\mathrm{s}}$ was measured by means of thermocouples. The obtained relation $\eta_{\mathrm{D}}\left(T_{\mathrm{s}}\right)$ is shown for both types of substrate in Fig. 10. It is quite satisfying to see that the evolution of the deposition rate with the substrate temperature is very similar for aluminium and copper plates although these two materials do not heat up at the same rate. In the measured range of temperatures the deposition rate appears to vary linearly; if this linear variation is extended to the whole temperature range a maximum deposition rate $(\xi=1)$ of about $26 \mathrm{~nm} / \mathrm{s}$ at $0 \mathrm{~K}$ is obtained and the total inhibition of the deposition would occur at a temperature of $1200 \mathrm{~K}$. 


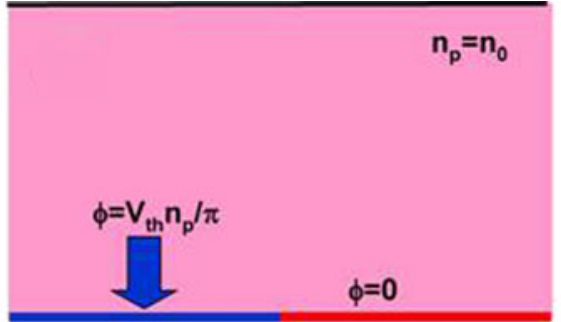

Pumping zone | Non-pumping zone
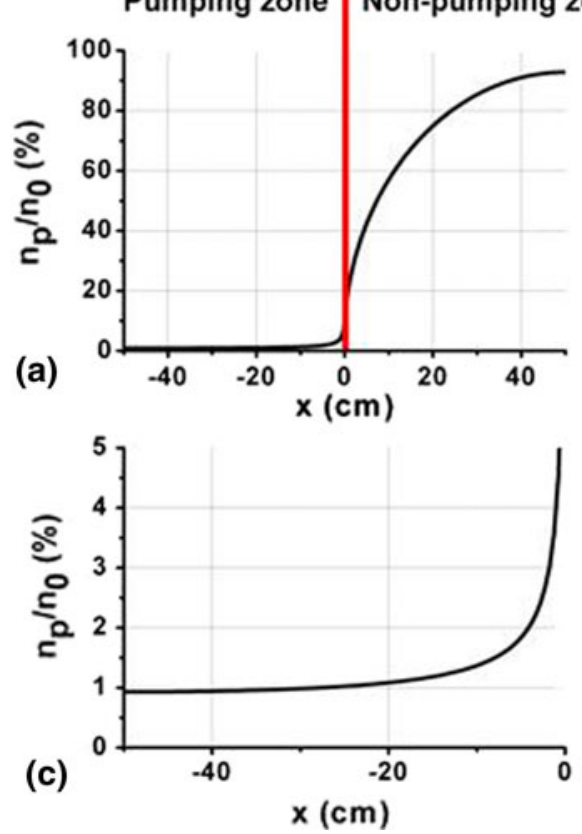

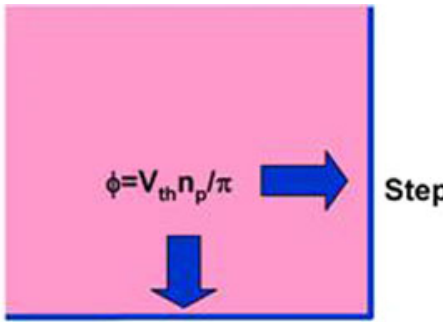

Substrate

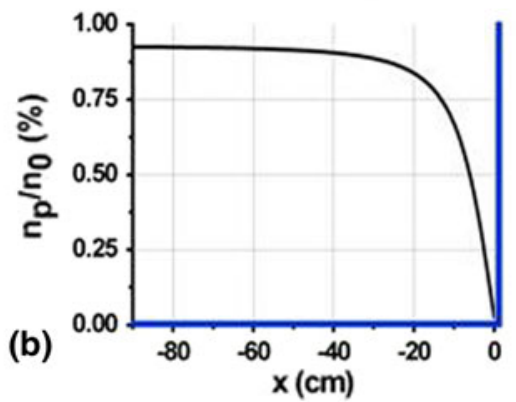

Fig. 8 Effect of precursor pumping discontinuity on the deposition profiles (a) whole normalized density profile for a semi-infinite pumping interface, (b) pumping corner, and (c) zoom on the deposition surface for semi-infinite pumping interface

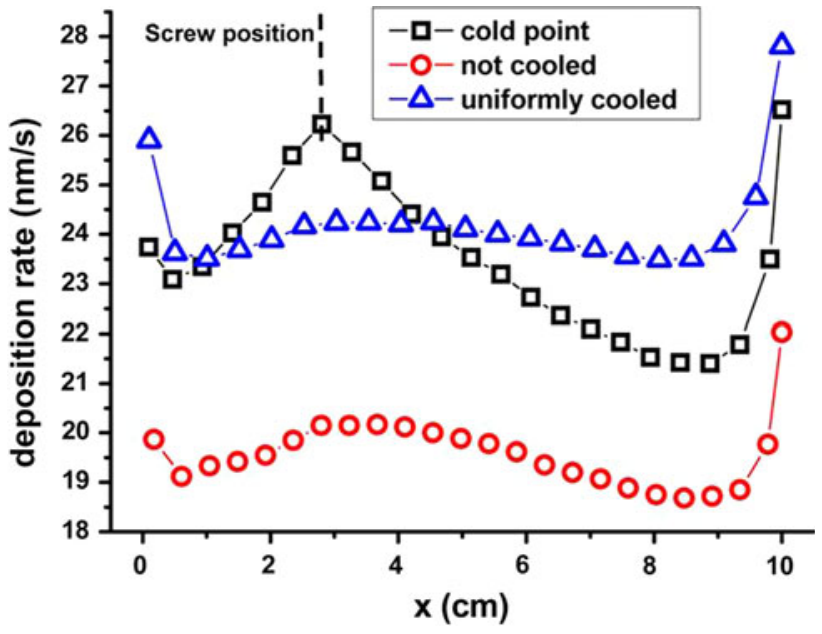

Fig. 9 Effect of the substrate cooling (not cooled, uniformly cooled and locally cooled) on the deposition rate

To account for a $26 \mathrm{~nm} / \mathrm{s}$ maximum deposition rate in the framework of the 1D convection-diffusion model exposed in the theoretical part of this article, the necessary precursor $(\mathrm{Si})$ normal flux at the confinement tube outlet

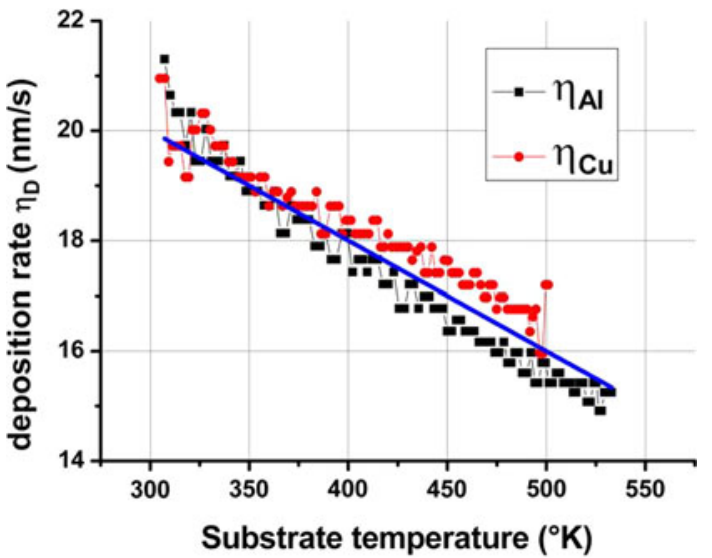

Fig. 10 Dependence of the deposition rate with the substrates temperature

has been estimated to be about $6-8 \times 10^{21} \mathrm{~m}^{-2} \mathrm{~s}^{-1}$. With an HMDSO evaporation rate of $30 \mathrm{~g} / \mathrm{h}$ and considering a full dissociation of the molecules a maximum Si flux at the tube outlet of about $3.5 \times 10^{21} \mathrm{~m}^{-2} \mathrm{~s}^{-1}$ can be expected. Furthermore the precursor losses on the internal surface of the confinement tube certainly lower this value 


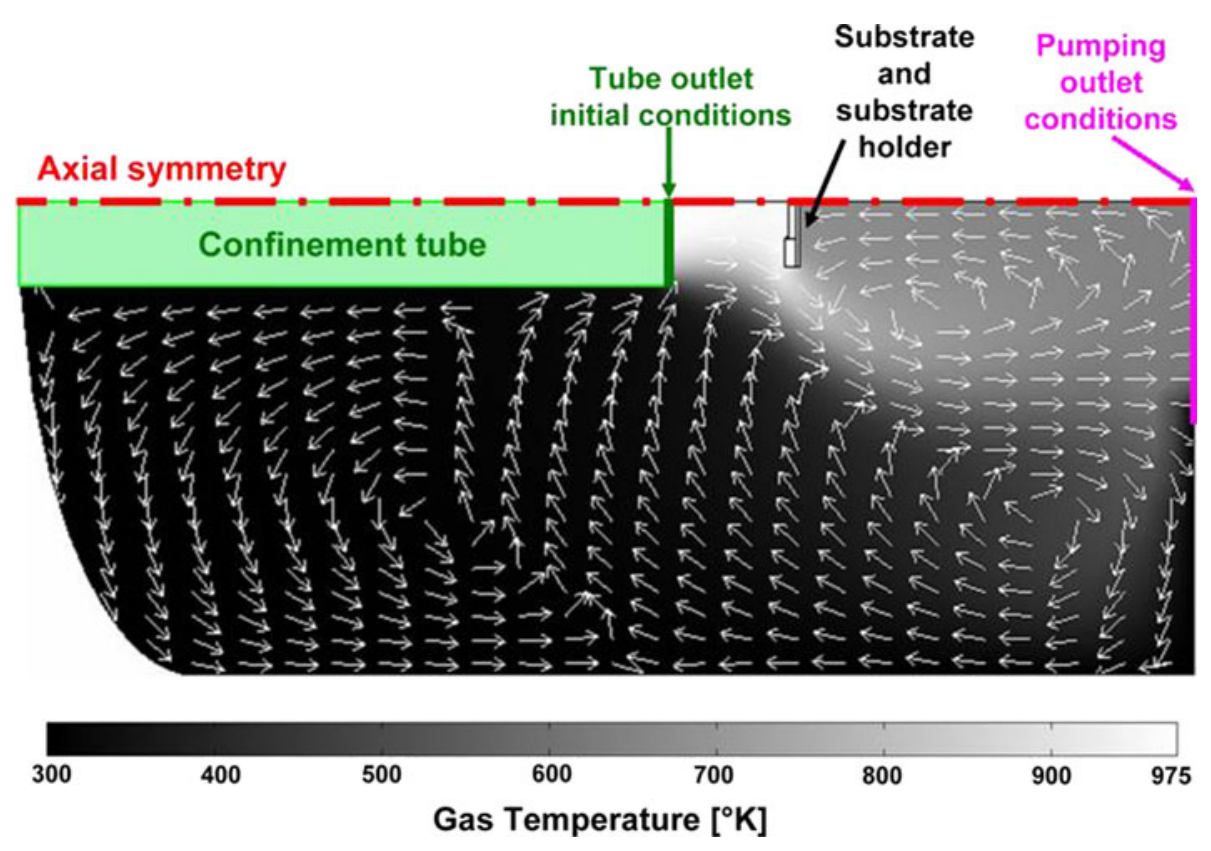

Fig. 11 COMSOL calculation of the gas temperature distribution and of the normalized fluid velocity field

non-negligibly. It then appears that the measured deposition rates are roughly two times higher than what would be expected at maximum. The reason for this discrepancy lies in the statement of a uniform constant pressure in the 1D model. On the contrary the impinging jet creates an over pressure region in front of the substrate and the precursor density must be enhanced as a consequence. Then to justify for the measured deposition rate the pressure on the substrate surface should be roughly two times higher than the pressure at the confinement tube outlet. To estimate the pressure increase on the substrate surface numerical solution of the compressible NavierStokes equation performed with COMSOL Multiphysics software (COMSOL, Inc.) has been used. The NavierStokes problem is also coupled to a conduction-convection resolution for the gas temperature. Figure 11 shows the typical geometry considered for these numerical resolutions: the process chamber is taken to be axially symmetric, the initial convective velocity profiles and gas temperature profiles are imposed at the confinement tube outlet according to the measured values for these parameters. The outlet conditions are given by the pumping which imposes a constant outlet pressure corresponding to the chamber pressure $(30 \mathrm{~Pa})$ and a convective transport of the heat. The image in Fig. 11 shows the calculated gas temperature distribution (grey scale) and the normalized fluid velocity field (arrows).

The pressure profile on the jet axis between the confinement tube outlet and the substrate is shown in Fig. 12 and it can be seen that a typically $65 \mathrm{~Pa}$ value is expected on the substrate surface while the pressure at the confinement tube outlet stays close to the $30 \mathrm{~Pa}$ of the chamber pressure.

Hence, once the over pressure created by the impinging jet is taken into account, our measured

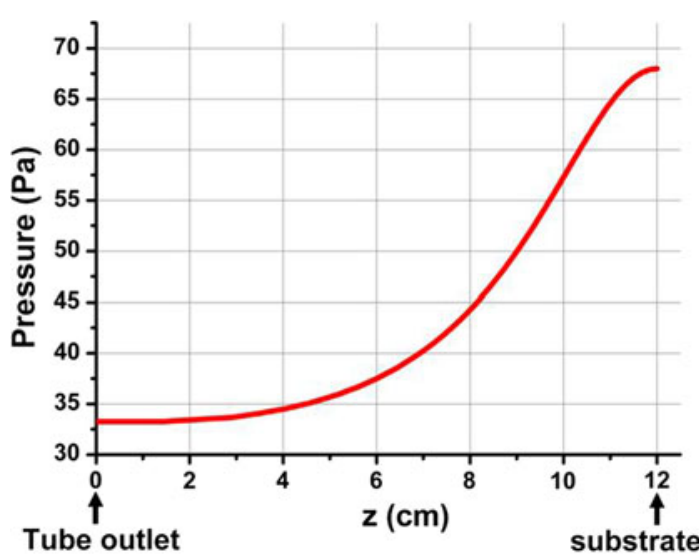

Fig. 12 COMSOL calculation of the pressure profile between the tube outlet and the substrate, taken on the jet axis

deposition rates are consistent with the theoretical approach provided that the injected HMDSO is very efficiently dissociated. This assumption is mainly sustained by our mass spectrometry measurements which show a total cancellation of the HMDSO cracking pattern, as well as of any molecule heavier than $44 \mathrm{amu}$ $\left(\mathrm{CO}_{2}\right)$, once the plasma is ignited.

The substrate temperature measurements can also be analysed in terms of the heat flux transferred from the plasma jet to the substrate. Considering first that the copper and the aluminium plates are small compared to the jet diameter, the heating of the substrates can be taken as uniform. Secondly we assume that the heat leaks can be neglected. This approximation is valid as long as the substrate temperature is far from its saturation value (typ. $900 \mathrm{~K}$ in our case). This being stated the heat flux received 


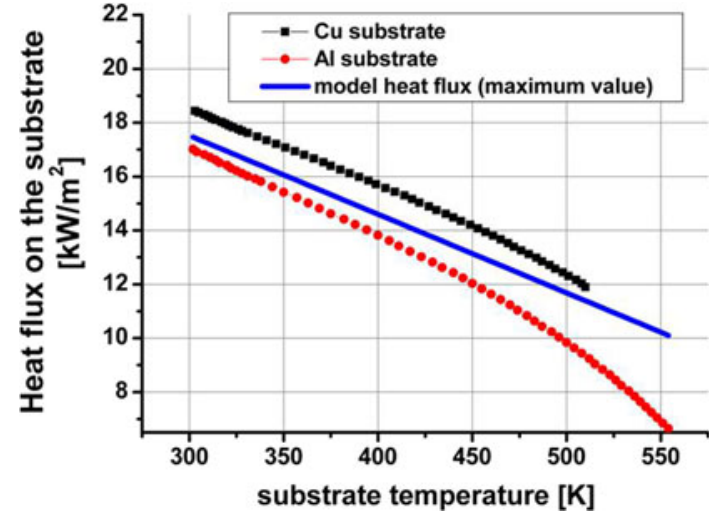

Fig. 13 Dependence with the substrate temperature of the heat flux transferred from the jet to the substrate. Dots: experimental; line: theoretical flux with a $65 \mathrm{~Pa}$ pressure on the substrate

by the substrates can be deduced from the time evolution of their temperature by using the following relation:

$$
Q=\rho_{\mathrm{s}} C_{\mathrm{s}} \mathrm{d} \frac{\mathrm{d} T}{\mathrm{~d} t}
$$

where $\rho_{\mathrm{s}}$ is the substrate mass density, $C_{\mathrm{s}}$ is the substrate specific heat and $d$ is the substrate thickness. With standard tabulated values for $\rho_{\mathrm{s}}$ and $C_{\mathrm{s}}$ the evolutions $Q\left(T_{\mathrm{s}}\right)$ shown in Fig. 13 are obtained. The solid line in Fig. 13 corresponds to the dependence of the heat flux expected according to our theoretical approach (with $\xi_{\mathrm{T}}=1$ ) and assuming a $65 \mathrm{~Pa}$ pressure on the substrate surface. The agreement with the experimental results is fairly good, especially at short times for which the assumption of negligible heat leaks is the more valid. It is interesting to note that a maximum value of the particles thermalization efficiency $\xi_{\mathrm{T}}$ has to be taken to account for our measurements.

\section{Conclusion}

The first conclusion arising from the present study concerns the use of a plasma torch at low pressures for PECVD processes. On the basis of simple theoretical considerations it has been shown that the high velocity of the plasma jet should not fundamentally affect the deposition mechanisms because these rely on diffusive processes on the substrate surface. In the framework of this approach the maximum deposition rate is determined by the precursor density and by the gas temperature on the interface, exactly as it would be for a conventional PECVD system, and the influence of the fluidic velocity is limited to the transport of the precursors, and of the heat, from the source to the substrate. On the other hand, 2D numerical calculations of the impinging jet problem show that the high velocity of the fluid is also responsible for an over pressure zone in front of the substrate and then leads to a consequent increase of the precursor density available for the deposition. All these contributions being taken into account the deposition rate can be estimated to be roughly 10 times higher for a $500 \mathrm{~m} / \mathrm{s}$ jet velocity than for purely diffusive transport. Once the over pressure zone in front of the substrate is taken into account, the deposition rates obtained in our $\mathrm{SiO}_{\mathrm{x}}$ coating experiments are consistent with the theoretical approach provided that we take the injected HMDSO to be almost fully dissociated.

Concerning the coatings uniformity two separate phenomena leading to non uniform deposition profiles were identified. The first one is related to the precursor pumping discontinuities which arises from the geometry of the substrate and of its environment. The second one is related to the local value of the sticking coefficient, which as an example was shown in this article to be dependent on the local substrate temperature.

Finally the expression of the heat flux transferred from the hot gas to the substrate appears to be quite accurate to justify for the measured evolutions of the substrate temperature. Satisfyingly the over pressure on the substrate surface has also to be introduced to account for the measured heat fluxes.

\section{References}

1. M.A. Lieberman and A.J. Lichtenberg, Principles of Plasma Discharges and Material Processing, Wiley, Hoboken, NJ, 1994

2. P. Fauchais, Understanding Plasma Spraying, J. Phys. D Appl. Phys., 2004, 37, p R86

3. T. Yoshida, The Future of Thermal Plasma Processing for Coating, Pure Appl. Chem., 1994, 66(6), p 1223-1230

4. M. Loch and G. Barbezat, Characteristics and Potential Application of Thermally Sprayed Thin Film Coatings, Thermal Spray: Surface Engineering via Applied Research, C. Berndt, Ed., ASM International, Material Park, OH, 2000, p 1141-1144

5. M. Gindrat, J.-L. Dorier, C. Hollenstein, M. Loch, A. Refke, A. Salito, and G. Barbezat, Effect of Specific Operating Conditions on the Properties of LPPS Plasma Jets Expanding at Low Pressure, Proceedings of the International Thermal Spray Conference 2002, E. Lugscheider, Ed., 4-6 March 2002 (Essen, Germany), 2002, p 459-464, DVS/IIW/ASM-TSS

6. A. Refke, G. Barbezat, J.-L. Dorier, M. Gindrat, and C. Hollenstein, Characterization of LPPS Processes Under Various Spray Conditions for Potential Applications, Thermal Spray 2003: Advancing the Science and Applying the Technology, International Thermal Spray Conference 2003, B.R. Marple and C. Moreau, Ed., 5-8 May 2003 (Orlando, CA, USA), ASM International, Materials Park, OH, 2003, 44073-0002

7. J.-L. Dorier, P. Guittienne, Ch. Hollenstein, M. Gindrat, and A. Refke, Mechanisms of Films and Coatings Formation from Gaseous and Liquid Precursors with Low Pressure Plasma Spray Equipment, Surf. Coat. Technol., 2009, 203, p 2125-2130

8. R.B. Bird, W.E. Stewart, and E.N. Lightfoot, Transport Phenomena, Wiley, Hoboken, NJ, 1960

9. R.J. Kee, M.E. Coltrin, and P. Glarborg, Chemically Reacting Flow, Wiley, Hoboken, NJ, 2003

10. M. Michaelidis and M. Pollard, Analysis of Chemical Vapor Deposition of Boron, J. Electrochem. Soc., 1984, 131, p 860-868

11. T. King-Ning, J.W. Mayer, and L.C. Feldman, Electronic Thin Film Science for Electrical Engineers and Materials Scientists, Macmillan, New York, 1992

12. J.-L. Dorier, M. Gindrat, Ch. Hollenstein, M. Loch, A. Refke, A. Salito, and G. Barbezat, Plasma Jet Properties in a New Process at Low Pressure for Large Area Thin Film Deposition, Thermal Spray 2001: New Surfaces for a New Millennium, C.C. Berndt, K.A. Khor, and E. Lugscheider, Ed., ASM International, Materials Park, OH, 2001, p 759-764 